\title{
Therapeutic approach of a case of rheumatoid arthritis complicated with renal amyloidosis in a young woman
}

\author{
Alexandra Burlui', Anca Cardoneanu1,2, Luana Macovei1,2, Claudia Banu², Elena Rezus ${ }^{1,2}$ \\ 1"Grigore T. Popa" University of Medicine and Pharmacy, Iasi, Romania \\ ${ }^{2}$ Clinical Rehabilitation Hospital, lasi, Romania
}

\begin{abstract}
Systemic AA amyloidosis is an uncommon complication of rheumatoid arthritis (RA), especially in poorly controlled RA. Renal involvement caused by deposits of amyloid fibrils derived from acute-phase proteins is represented by nephrotic-range proteinuria ultimately leading to renal failure. We report the case of 27 -year-old woman diagnosed with RA, with a history of musculoskeletal involvement since the age of 16 , manifested by arthritis involving the wrists, knees and ankles; accompanied by severe and persistent inflammatory syndrome. She presented with an active form of RA complicated with secondary amyloidosis manifested by nephrotic-range proteinuria at the time of admission. Renal biopsy confirmed the presence of amyloid deposits. Anti-TNFa therapy with Certolizumab pegol proved effective in reducing proteinuria and inflammatory biomarker levels in our patient. The efficacy of anti TNF- $\alpha$ therapy in RA complicated with renal amyloidosis is demonstrated by recent scientific data.
\end{abstract}

Keywords: amyloidosis, proteinuria, rheumatoid arthritis, certolizumab pegol

\section{CASE REPORT}

We report the case of a 27 -year-old female with a history of Juvenile Idiopathic Arthritis since the age of 16. As an adult, she was diagnosed with Rheumatoid Arthritis in March 2012 according to ACR criteria (American College of Rheumatology) and followed various treatment regimens with conventional DMARDs (methotrexate, leflunomide, sulfasalazine) with poor therapeutic response and progressively severe joint involvement. In March 2012 she presented with inflammatory pain and swelling in the right knee, right ankle and wrists, accompanied by morning stiffness. Physical examination revealed joint swelling and tenderness in both ankles, right knee and wrists as well as edema in the lower legs.

Routine laboratory findings revealed the presence of systemic inflammation (erythrocyte sedimentation rate, $\mathrm{ESR}=126 \mathrm{~mm} / \mathrm{h}$ and C-reactive protein, $\mathrm{CRP}=79.45 \mathrm{mg} / \mathrm{l})$, hypoproteinemia (33 g/l), proteinuria $(3.7 \mathrm{~g} / 24 \mathrm{~h})$. However, serum creatinine, uric acid and creatinine clearance were within normal limits. Immunological investigations showed rheumatoid factor positivity $(47.2 \mathrm{U} / \mathrm{ml})$, antibodies to citrullinated peptide antigens within normal limits $(1.9 \mathrm{U} / \mathrm{ml})$, and negative antinuclear antibodies. Hand X-rays revealed severe changes of bilateral wrists (erosions, ankylosis), erosions and moderate narrowing of joint space at the bilateral 2 and 3 metacarpophalangeal joints.

In order to identify the causative factor of our patient's proteinuria, she was referred to the Nephrology Clinic where a renal biopsy was prelevated. The specimen proved positive for amyloidosis on Congo red staining. Consequently, the patient was started on immunosuppressive therapy with Cyclosporine and glucocorticoids (a short course of iv methylprednisolone, then Prednisone, $10 \mathrm{mg} /$ day with slow taper) without significant improvement after two months. We initiated Cyclophosphamide pulse therapy $(1,000 \mathrm{mg} / \mathrm{month}$, total dose of 4,000 mg). The patient also received anti-proteinuric treatment (Quinapril, $2.5 \mathrm{mg} /$ day) and diuretic therapy (Furosemide, $40 \mathrm{mg}$ /day). While under Cyclophosphamide, CRP levels decreased but high ESR values, as well as proteinuria $(4.8 \mathrm{~g} / 24 \mathrm{~h})$ and hypoproteinemia $(35 \mathrm{~g} / \mathrm{l})$ remained present (Fig. 1). 

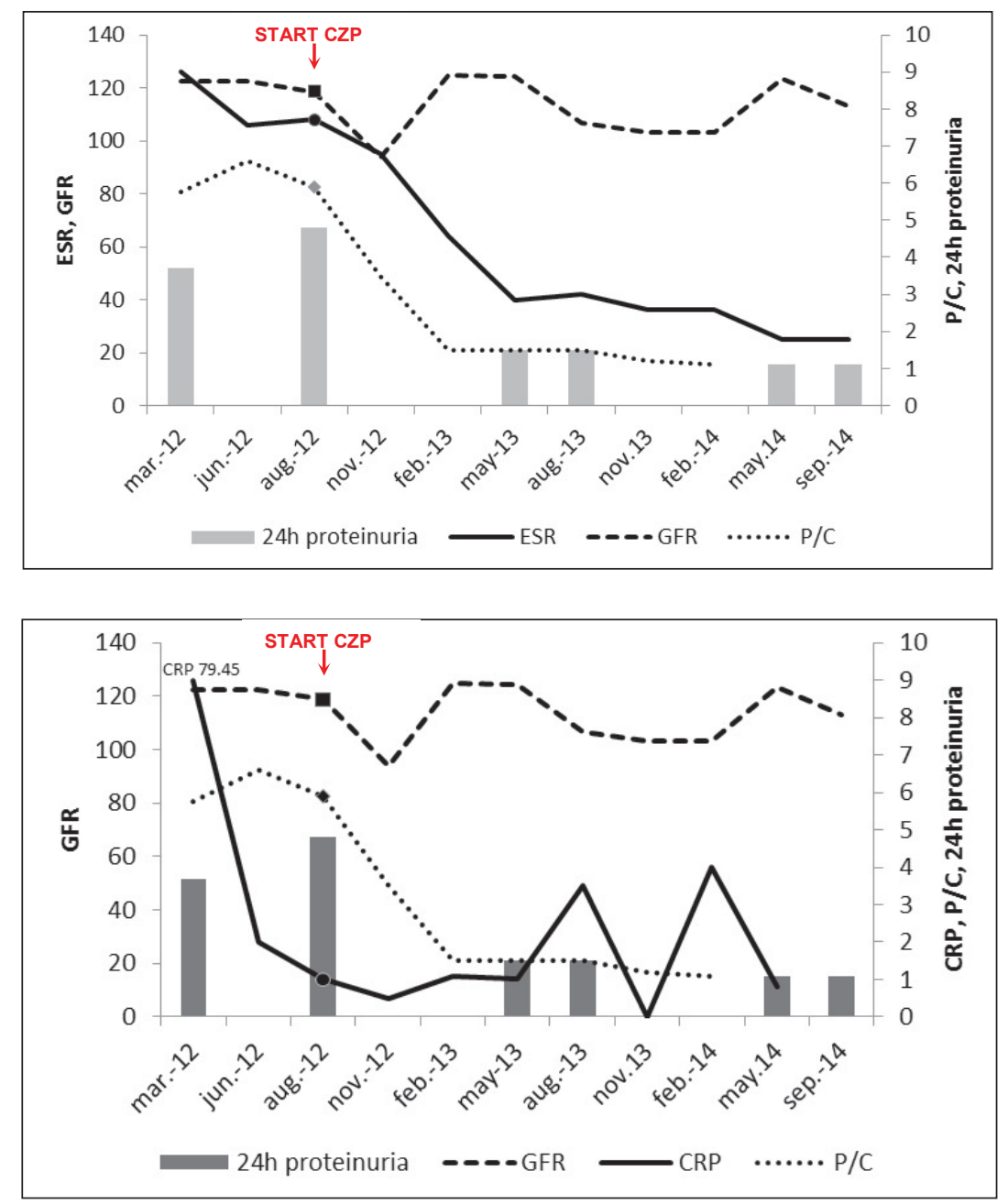

FIGURE 1. Laboratory findings between March 2012 - September 2014. $E S R$ - erythrocyte sedimentation rate; GFR - glomerular filtration rate; $P / C$ - protein/creatinine ratio; $C R P-C$ reactive protein.

Poor therapeutic response to conventional DMARDs motivated the initiation of biological therapy with TNF-alpha inhibitor Certolizumab pegol in association with Cyclosporine, $150 \mathrm{mg} /$ day. The patient followed Certolizumab pegol (CZP) treatment $200 \mathrm{mg} \times 2$ subcutaneous injections every two weeks for the first three administrations (weeks 0, 2 and 4), then $200 \mathrm{mg}$ subcutaneously every two weeks.

We observed a significant decrease in inflammatory marker levels, improvement of proteinuria and total serum protein $(55 \mathrm{~g} / \mathrm{l})$, as well as preserved kidney function after one year of Certolizumab pegol therapy (Figure1). Following two years of anti-TN$\mathrm{F} \alpha$ therapy, the patient achieved remission demonstrated by disease activity score values (DAS28 $=2.6$ ) (Figure 2). Furthermore, a decrease in proteinuria was observed (1.1 g/24 h). Serum creatinine, creatinine clearance and uric acid levels remained within normal limits, while total serum protein concentrations increased (60 g/l) (Fig. 1).

\section{DISCUSSIONS}

Systemic amyloidosis is a potential complication of several chronic inflammatory diseases like RA, ankylosing spondylitis and Crohn's disease. AA amyloidosis represents one of the most severe complications of RA $(1,2)$. Among chronic inflammatory diseases, RA is the most frequently associated with AA amyloidosis (33\%) (3). Tissue damage results from extracellular deposition of insoluble AA amyloid fibrils derived from the circulatory acute-phase reactant precursor serum amyloid A protein (SAA). SAA concentration is strongly correlated with disease activity (4). SAA is produced in hepatocytes following stimulation by proinflammatory cytokines tumor necrosis factor alpha (TNF $\alpha$ ). AA amyloid deposits are predominantly found in the kidney, liver, spleen and gastrointestinal tract $(5,6)$.

The incidence of AA amyloidosis after 15 years of RA is estimated to be found in $10 \%$ of patients (7). The diagnosis of AA amyloidosis requires histo- 


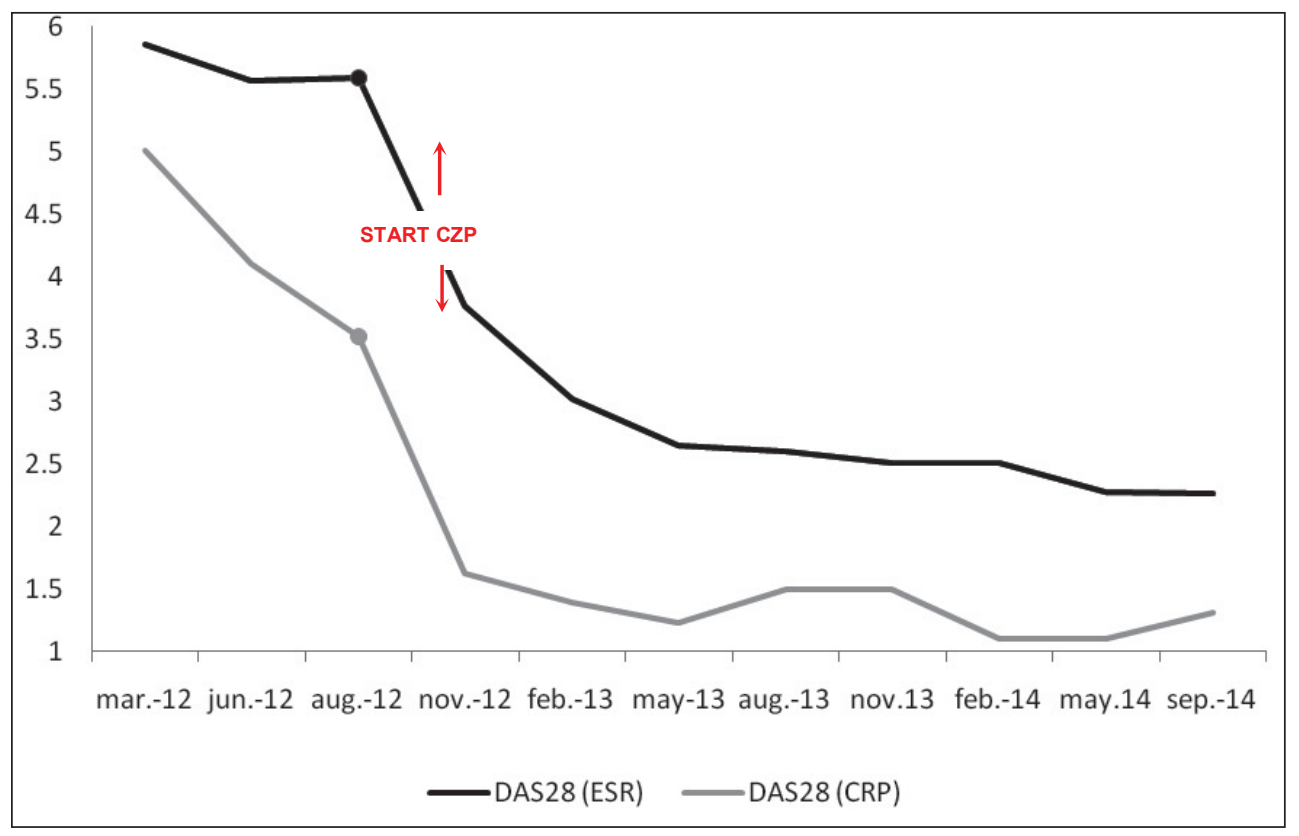

FIGURE 2. Evolution of disease activity score DAS28.

logical examination in order to demonstrate the presence of amyloid deposits $(2,6)$. Biopsies can be also obtained from the involved organs, such as kidney, liver or gastrointestinal tract $(7,8)$. A significant correlation was found between renal function parameters and the area of AA amyloid deposits in renal tissue specimens $(9,10)$. Glomerular deposition is associated with a poorer prognosis than vascular and tubular amyloid deposition in RA patients with AA amyloidosis (11).

In RA complicated with renal AA amyloidosis, the therapeutic goal is to achieve strict suppression of inflammation (low values of CRP). It was proven that SAA concentration decrease is correlated with significant improvement of kidney function and stabilization of AA deposits, which also prolong survival $(6,12)$. Risk factors associated with poor prognosis in AA amyloidosis include low serum albumin levels and high CRP concentrations throughout follow-up (13).

The aim of AA amyloidosis therapy is to achieve optimal control of the underlying disease. Cytotoxic therapy such as $\mathrm{CYC}$, as well as anti TNF $\alpha$ and anti IL-6 agents were reported effective in treating both RA and AA amyloidosis $(3,14)$. A retrospective study revealed that pulse therapy with CYC is able to reduce the risk of end-stage renal failure and increase survival (15). It is suggested that the use of immunosuppressive agents may improve prognosis, and $\mathrm{CYC}$ was proven superior to methotrexate
(MTX) in treating RA-related AA amyloidosis (16). However, systemic inflammation as well as proteinuria were persistent under treatment with cyclophosphamide in our patient.

Clinical studies have shown the efficacy and safety of certolizumab pegol in active RA (17). We initiated anti-TNF $\alpha$ therapy with CZP in association with Cyclosporine which ultimately led to clinical resolution of the nephrotic syndrome. Treatment with CZP slowed down the progression of AA amyloidosis by reducing proteinuria and decreasing systemic inflammation.

Our patient did not experience clinical improvement under immunosuppressive treatment (Cyclophosphamide, Cyclosporine, corticosteroids). Futhermore, both inflammatory biomarkers and proteinuria were persistently high. Nonetheless, anti-TNF $\alpha$ therapy with Certolizumab pegol in association with Cyclosporine proved effective in reducing proteinuria and induced remission in our patient.

\section{CONCLUSIONS}

AA amyloidosis is an uncommon complication of RA. Patients with RA develop AA amyloidosis especially in poorly controlled disease, renal failure being the most common cause of mortality. Treatment of RA-related AA amyloidosis focuses on suppressing the underlying inflammatory condition. The efficacy of anti-TNF- $\alpha$ therapy in the treatment 
of RA complicated with renal amyloidosis was demonstrated by current scientific data. Aggressive therapeutic interventions including biological drugs for optimal control of the inflammatory process associated with RA have the potential to influence the evolution of renal AA amyloidosis.

Conflict of interest: none declared Financial support: none declared

\section{REFERENCES}

1. Obici L., Raimondi S., Lavatelli F, et al. Susceptibility to AA Amyloidosis in Rheumatic Diseases: A Critical Overview. Arthritis\&Rheumatism. 2009 Oct,61(10): 1435-1440.

2. Fernandez I.M., Rodrigues A.M., Perez S.G. Use of Etanercept in Amyloidosis Secondary to Rheumatoid Arthritis: A Report of Two Cases. Reumatol Clin. 2011; 7(6):397-400.

3. Ueno T., Takeda K., Nagata M. Remission of proteinuria and preservation of renal function in patients with renal AA amyloidosis secondary to rheumatoid arthritis. Nephrol Dial Transplant. 2012; 27:633-639.

4. Gottenberg J.E, Vincent F.M, Bentaberry F. Anti-tumor Necrosis Factor Therapy in Fifteen Patients With AAAmyloidosis Secondary to Inflammatory Arthritides. Arthritis\&Rheumatism. 2003 Jul, 48(7): 2019-2024.

5. Nakamura T. Clinical strategies for amyloid $A$ amyloidosis secondary to rheumatoid arthritis. Modern Rheumatology. 2008, 18:109-118.

6. Real de Asua D., Costa R. et al. Systemic AA amyloidosis: epidemiology, diagnosis, and management. Clinical Epidemiology. 2014:6 369-377.

7. Nobre C.A. et al. Anti-TNF therapy in renal amyloidosis in refractory rheumatoid arthritis: a new therapeutic perspective. Bras J Rheumatol. 2010; 50 (2):205-210.

8. Perfetto F., Moggi-Pignone A., et al. Systemic amyloidosis: a challenge for the rheumatologist. Nat Rev. Rheumatol. 2010, 6:417-429.

9. Kuroda T., Wada Y., et al. Effective anti-TNF- $\alpha$ therapy can induce rapid resolution and sustaine decrease of gastroduodenal mucosal amyloid deposits in reactive amyloidosis associated with rheumatoid arthritis. J Rheumatol. 2009, 36:2409-2415.
10. Kuroda T., Tanabe N., Kobayashi D. et al. Significant association between renal function and area of amyloid deposition in kidney biopsy specimens in reactive amyloidosis associated with rheumatoid arthritis. Rheumatology International. 2012 Oct, 32(10): 3155-3162.

11. Bergesio F., Anna Maria Ciciani A.M., Santostefano M. Renal involvement in systemic amyloidosis - an Italian retrospective study on epidemiological and clinical data at diagnosis. Nephrol Dial Transplant. 2007, 22: 1608-1618.

12. Lachmann H.J., Goodman H.J., Gilbertson J.A. Natural history and outcome in systemic AA amyloidosis. N Engl J Med. 2007; 356:2361-2371.

13. Joss N., McLaughlin K., Simpson K., et al. Presentation, survival and prognostic markers in AA amyloidosis. Q J Med. 2000; 93:535-542.

14. Nakamura T., Higashi S. Et al. Effectiveness of etanercept vs cyclophosphamide as treatment for patients with amyloid $A$ amyloidosis secondary to rheumatoid arthritis. Rheumatology. 2012, 51:2064-2069.

15. Chevrel G., Jenvrin B.C., McGregor B., Miossec P. Renal type AA amyloidisis associated with rheumatoid arthritis: a cohort study showing improved survival on treatment with pulse cyclophosphamide. Rheumatology. 2001, 40:821-825.

16. Nakamura T., Higashi, S. et al. Significance of SAA1.3 allele genotype in Japanese patients with amyloidosis secondary to rheumatoid arthritis. Rheumatology (Oxford), 2006 Jan, 45: 43-49.

17. Smolen J., Landewe R.B. et al. Efficacy and safety of certolizumab pegol plus methotrexate in active rheumatoid arthritis: the RAPID 2 study. A randomised controlled trial. Ann Rheum Dis. 2009; 68:797-804. 